Meta

Journal des tradlucteurs

Translators' Journal

\title{
Platerm : la banque de terminologie du système Platon
}

\section{Wallace Schwab et Richard St-Denis}

Volume 25, numéro 3, septembre 1980

URI : https://id.erudit.org/iderudit/004061ar

DOI : https://doi.org/10.7202/004061ar

Aller au sommaire du numéro

Éditeur(s)

Les Presses de l'Université de Montréal

ISSN

0026-0452 (imprimé)

1492-1421 (numérique)

Découvrir la revue

Citer cet article

Schwab, W. \& St-Denis, R. (1980). Platerm : la banque de terminologie du système Platon. Meta, 25(3), 287-302. https://doi.org/10.7202/004061ar

\section{Résumé de l'article}

Les auteurs présentent une description des éléments et du fonctionnement de la banque de terminologie PLATERM. Ils évoquent aussi certaines notions contemporaines de terminologie et de lexicographie qui ont servi de base à la réalisation de la banque. Enfin, ils fournissent un aperçu de l'usage fait de PLATERM depuis sa mise en opération. 


\section{Platerm: la banque de terminologie du système Platon}

Wallace Schwab

RICHARD ST-DENIS

\section{RÉSUMÉ}

Les auteurs présentent une description des éléments et du fonctionnement de la banque de terminologie PLATERM. Ils évoquent aussi certaines notions contemporaines de terminologie et de lexicographie qui ont servi de base à la réalisation de la banque. Enfin, ils fournissent un aperçu de l'usage fait de PLATERM depuis sa mise en opération.

Mots clés : banque de terminologie, système PLATON, traduction, terminologie, système interactif.

\section{INTRODUCTION}

Le présent article a pour objectif la description de l'implantation et du fonctionnement d'une banque de terminologie utilisée exclusivement en milieu interactif et développée spécifiquement sur le système PLATON.

Aux États-Unis, PLATO (Programmed Logic for Automated Teaching Operation), est le nom utilisé pour désigner un programme de recherches lancé en 1959 au Coordinated Science Laboratory de l'Université de l'Illinois, sous la direction de Donald Bitzer, dans le but d'étudier l'utilité de l'informatique dans le processus d'apprentissage ${ }^{1}$. Au Québec, et plus précisément à l'Université du Québec, on préfère se servir du nom PLATON.

Le système PLATON est avant tout un moyen de communication qui permet aux médiatiseurs ${ }^{2}$, aux professeurs et aux étudiants de créer, diffuser et recevoir de l'information. En tant que système informatisé d'apprentissage,

1. D.L. Bitzer et al, Computer-based science education, CERL Report X-37, Computer-based Education Research Laboratory, University of Illinois, Urbana, 1973.

2. Médiatiseur: usager qui transcrit en langage PLATON une leçon, un contenu de communication. 
PLATON donne une formation individualisée grâce à la présentation de leçons préparées en fonction des besoins de chaque étudiant.

Le langage informatique utilisé par ce système, qu'on appelle langage PLATON, ou langage de médiatisation, ou encore langage médiatique, permet la programmation d'éléments de cours sans avoir à recourir à un spécialiste de la programmation. Grâce à PLATON, l'étudiant a la possibilité, à l'aide d'un terminal, de consulter une leçon pédagogique qui lui offre des expériences d'apprentissage telles que l'exercice répété, la démarche tutorielle, l'enquête, la simulation, les jeux éducatifs et le dialogue ${ }^{3}$. Voilà donc le cadre général dans lequel se situe le présent exposé sur la banque de terminologie PLATERM.

\section{Platerm}

On désigne sous le nom de PLATERM le système de gestion de la banque de terminologie qui contient le vocabulaire bilingue du système PLATON. Les principales caractéristiques de cette banque sont la mise à jour continue des données, la consultation en direct par l'usager et la gestion informatisée. Pour répondre aux besoins de ses usagers, PLATERM fournit des réponses efficaces et rapides, le tout situé dans le cadre d'une banque de taille moyenne. Enfin, l'avantage le plus évident de PLATERM provient de sa capacité de donner au traducteur le mot juste, la précision textuelle grâce à l'uniformisation de la terminologie et un centre documentaire fondé sur la bibliographie informatisée.

Par le truchement d'un réseau de communication, celui de l'Université du Québec qui s'étend sur une superficie de centaines de kilomètres, on met à la disposition de l'ensemble de la clientèle des constituantes de l'Université du Québec une banque de terminologie informatisée. Ainsi, PLATERM trouve place parmi les systèmes avant-gardistes et, de ce fait, se distingue des moyens plus traditionnels comme les dictionnaires et les ouvrages imprimés.

L'origine anglo-américaine du système PLATON a entraîné le développement de PLATERM. En effet, un usager désireux de se servir en français du système PLATON doit s'adapter à un outil conçu par et pour un autre groupe ethnique dont les réflexes culturels sont parfois fort différents. C'est ainsi que la traduction et, dans la plupart des cas, l'acclimatation culturelle des leçons sont de rigueur pour rendre le système utilisable en français. Selon son objectif, la banque contient les données dont un traducteur ou un rédacteur pourrait avoir besoin pour accomplir sa tâche. À titre d'exemple, on sait que le besoin le plus immédiat d'un traducteur francophone consiste à connaître l'équivalent français des termes techniques anglais qu'il rencontre. Par ailleurs, pour un terme peu usité, il pourrait désirer des renseignements complémentaires comme une définition ou un exemple de bon usage. Or, si la banque ne contient pas ce que cherche le traducteur, il voudra peut-être consulter la bibliographie informatisée ou même laisser, dans le fichier réservé aux messages à l'intention du responsable du système, un petit mot expliquant son problème.

3. Pour des renseignements détaillés sur le système PLATON, consulter: PLATON, un système multimédia d'apprentissage, information générale 2, publication no Pl.2A, Université du Québec,
Québec, 1977 . 
PLATERM résulte des recherches conduites par le Comité de terminologie ${ }^{4}$ de l'Université du Québec, groupe pluridisciplinaire formé dans le but de découvrir, d'analyser et de proposer aux usagers du système américain PLATO une bonne terminologie française. Pour atteindre le but ultime de ces recherches, soit l'établissement et le maintien d'une banque de terminologie, on a eu recours davantage à une enquête lexicographique qu'aux méthodes usuelles de la terminologie contemporaine.

Pour mieux situer ces propos, il est utile de rappeler les idées de Robert Dubuc $^{5}$, qui ont servi de fondement théorique aux travaux du comité et expliquent la démarche fondamentale suivie en terminologie de même qu'en lexicographie.

D'une part le lexicographe, préoccupé par le décodage du message, pose une question comme: «Que signifie un terme X?». D'autre part le terminologue, saisi du vocabulaire en situation, est porté à poser des questions du genre: «Comment appelle-t-on l'objet qui...?» ou «Comment désigne-t-on l'opération qui consiste à...?». De même, Dubuc explique que le mot «terminologie» couvre une réalité à deux facettes :

1) Traditionnellement, la terminologie est l'ensemble de tous les termes propres à une activité ou à une discipline.

2) Plus récemment, elle désigne le processus d'organisation ou de groupement des termes qui appartiennent à cette activité ou à cette discipline.

On peut donc en déduire qu'en terminologie, on favorise la recherche et l'inventaire du vocabulaire en situation, processus qui consiste alors à identifier des concepts.

Cette démarche va au-delà de la recherche de définition des notions rudimentaires et permet de découvrir tous les moyens d'expression dans un domaine donné. Enfin, toujours selon Dubuc, une bonne collection en terminologie doit répondre fidèlement aux besoins de l'usager.

En plus de s'inspirer des principes précédents, PLATERM se fonde sur un développement empirique: définition et catalogage des mots et des concepts, même rudimentaires (démarche surtout lexicographique), description des usages, identification des notions et stylistique (démarche avant tout terminologique). La première étape dans l'élaboration de PALTERM a consisté à établir une base de données, ce qui s'est accompli en regroupant les documents les plus importants sur le sujet ${ }^{6}$.

4. Sous la direction de monsieur Pierre Dumas, directeur du développement, vice-présidence aux communications, le comité est composé de Jean Beaudot, ingénieur, linguiste et mathématicien, Rachel Chouinard, traductrice, Robert Dupuy, mathématicien et physicien, René Lortie, agent de recherche, Joseph Reid, physicien, Claude Rigault, pédagogue, Wallace Schwab, traducteur.

5. R. Dubuc, Manuel pratique de terminologie, p. 14.

6. CDC, Plato Author Language Reference Manual, Publication No. 97405100, Control Data Corporation, St. Paul, Minnesota, 1977.

CDC, Plato System Overview, Publication No. 97406700, Control Data Corporation, St. Paul, Minnesota. 1977.

CDC, Plato User's Guide, Publication No. 97405900, Control Data Corporation, St. Paul, Minnesota, 1976.

J. Ghesquière, C. Davis et C. Thompson, Institution to TUTOR. Computer-based Education Research Laboratory, University of Illinois, Urbana, 1974. 
Des passages pertinents de ces documents ont été mis en mémoire et il a été possible par la suite de faire une partie du dépouillement terminologique à l'aide des listes KWIC (Key Work in Context). Les membres du comité ont examiné chaque document et ils ont retenu sur fiches la terminologie pertinente ainsi que les traductions suggérées. La deuxième étape a comporté, bien entendu, l'analyse des données et la sélection des solutions et la dernière étape, la création d'une «leçon PLATON» (programme rédigé en langage médiatique) pour maîtriser le maniement des fiches.

Chaque fiche ou entrée contient un ensemble de renseignements sur un terme donné; on y trouve notamment:

1. Le terme: l'accès primaire à l'information se fait en demandant un terme (maximum 60 caractères).

2. La traduction: on peut compter jusqu'à neuf traductions pour un terme et, comme pour le terme, chaque traduction peut utiliser un maximum de 60 caractères.

3. La définition: une description précise, claire et concise du contenu notionnel de l'unité lexicale figure dans cette case.

4. Le contexte ou l'exemple: selon Robert Dubuc ${ }^{7}$, un bon contexte correspond à l'un des trois types suivants:

4.1 le contexte définitoire,

4.2 le contexte explicatif,

4.3 le contexte associatif.

Dans PLATERM, en anglais, on retrouve tous les contextes dans l'une de ces trois catégories. En français, on trouve des exemples au lieu de contextes. Suivant rigoureusement le principe de terminologie voulant que les textes traduits soient exclus, il n'est pas possible d'avoir en français de véritables contextes. Cette démarche découle du fait que la majeure partie de la version française résulte de la traduction de documents américains. La mission du comité de terminologie est de conseiller aux usagers du système des emplois de mots corrects et uniformes, ce qui crée un dilemme. Le comité a décidé qu'en anglais, on recourrait aux bons contextes et qu'en français, des exemples seraient soumis aux membres pour approbation. Ensuite, l'exemple acquerrait valeur de contexte «officieux».

5. Les notes: cette case résulte d'un désir de fournir à l'usager diverses données complémentaires au sujet du terme recherché. Par exemple, on trouve beaucoup de notes où les mots associés sont indiqués (sous le terme «grille», on réfère le lecteur à "grille fine» ou à "grille large»). Ou bien, d'autres notes mettent en garde contre les usages ambigus ou erronés.

Dans l'ensemble de la banque, pour authentifier chaque sens donné, on en indique les sources sous forme d'abréviations encadrées de parenthèses. Si l'usager désire en savoir davantage sur une référence, il peut inscrire l'abréviation au bas de son écran, ce qui déclenche l'affichage automatique du titre de l'ouvrage désigné. Au cas où l'usager voudrait encore plus de renseignements,

7. Op. cit., p. 30. 
la consultation des pages de bibliographie lui permettra de prendre connaissance des informations bibliographiques habituelles. En outre, deux mises en garde automatiques peuvent, à l'occasion, apparaître sur l'écran pour avertir l'usager d'une difficulté particulière, ou d'un point d'intérêt, que ce soit dans la définition, l'exemple, les notes ou la traduction.

À l'aide des statistiques que compile PLATERM, il est possible de repérer en un clin d'œil le cheminement des usagers. Le recours aux statistiques et aux commentaires des usagers est l'instrument par excellence dont dispose le responsable de PLATERM pour l'interprétation de l'action-réaction entre le système et ses usagers.

Bien d'autres recours sont accessibles à l'usager pour comprendre le fonctionnement de la banque ou les multiples aspects du système PLATON.

1. Aides: on peut demander au système de l'information relative à son fonctionnement ou à celui d'un dispositif particulier de la banque.

2. Les commentaires : cette section est un fichier à l'intention des usagers qui veulent faire savoir au responsable ce qu'ils pensent d'un aspect quelconque de la banque. Tout usager peut lire et enregistrer des commentaires.

3. La bibliographie: en recherche terminologique, il est un principe fondamental selon lequel on doit TOUJOURS indiquer la source d'un renseignement. Par le biais du fichier bibliographique, les données référentielles font partie intégrante du système.

4. La liste des cónsultants: toutes les personnes ressources sont inscrites dans la liste des consultants.

\section{Platon}

La banque de terminologie fonctionne grâce à une infrastructure dont les principaux éléments sont mis en relief dans les lignes qui suivent.

Les deux composantes indispensables au fonctionnement du système PLATON sont le matériel et le logiciel qui en commande l'ensemble. Le matériel se compose d'un ordinateur CDC Cyber 73, d'une mémoire électronique auxiliaire, d'une mémoire de masse, de liens de communications et de terminaux PLATON. Le logiciel comprend le système d'exploitation ${ }^{8}$, le système en temps partagé PLATO et le langage de médiatisation PLATON.

L'usager utilise le terminal PLATON pour interroger PLATERM. Les principaux éléments du terminal PLATON sont un écran et un clavier. Le clavier constitue l'organe principal pour l'entrée des données dans le système PLATON. Les touches servant à inscrire les différents caractères ressemblent beaucoup à celles d'une machine à écrire ordinaire et, de plus, on retrouve sur le clavier des touches fonctions qui permettent à l'usager d'exécuter des opérations particulières. Par exemple, à la demande de l'usager, la touche HELP fournira des informations relatives au fonctionnement de la banque de terminologie. Une fois que l'usager a obtenu les informations désirées, il peut revenir à son

8. NOS (Network Operating System). 
point de départ en appuyant sur la touche BACK. En outre, en appuyant sur la touche LAB, l'usager commande au système de passer de la banque française à la banque anglaise et vice versa. Les informations apparaissent sur l'écran sous forme de textes ou autres signes (figures, chiffres, codes, etc.). L'écran peut présenter simultanément jusqu'à 32 lignes horizontales de caractères alphanumériques et chacune d'elles peut contenir 64 caractères. Le taux de transfert des caractères est suffisamment rapide pour afficher un texte de longueur moyenne en moins de six secondes. De plus, la structure du système PLATON est équilibrée de façon à assurer à l'usager un délai de réponse de l'ordre de 0,2 seconde, quel que soit le nombre de terminaux en usage.

\section{LE FONCTIONNEMENT}

À l'heure actuelle, toutes les données traitées par PLATERM sont groupées dans onze fichiers à accès direct, conservés dans la mémoire de masse. Un premier fichier comporte les commentaires, le nom des membres, la bibliographie, la liste des consultants, la note générale et divers paramètres affectés au bon fonctionnement de la banque. Deux autres fichiers contiennent respectivement la liste des termes anglais et celle des termes français, rangés par ordre alphabétique dans la banque. Ces fichiers s'avèrent nécessaires lorsqu'un usager désire consulter la liste des termes. Les autres fichiers sont divisés en deux groupes comprenant chacun les fiches anglaises et les fiches françaises. Dans sa forme actuelle, PLATERM est conçu comme un dictionnaire spécialisé et, de ce fait, ne contient que 712 termes en anglais et 712 termes en français, ce qui est amplement suffisant pour satisfaire les besoins courants. Un usage constant de PLATERM accroîtra inévitablement la demande pour ce genre d'information et nécessitera éventuellement une expansion de la banque. Aujourd'hui, la capacité de PLATERM peut être rapidement portée à 2848 termes par le simple ajout de fichiers et par une mise à jour des pointeurs.

Pour gérer efficacement la banque, PLATERM distingue quatre types d'usagers: le coordinateur, le responsable, les membres et les usagers ordinaires. Le coordinateur nomme le responsable et les membres; il peut limiter l'accès aux banques pour n'importe qui.

Le responsable est chargé de la mise à jour des banques française et anglaise et prépare également tout le matériel auquel les usagers ont accès. Les usagers ordinaires et les membres ont accès aux banques française et anglaise ainsi qu'aux listes de termes. Ils peuvent aussi enregistrer ou lire des commentaires, et consulter la bibliographie et la liste des consultants. La différence entre un usager ordinaire et un membre réside dans le fait que les membres sont inscrits. Cette distinction permet d'accorder ou de retirer des privilèges à certaines catégories d'usagers.

Le système affiche différentes pages sur l'écran et généralement, à chaque page, il pose une question à l'usager. De plus, il affiche au bas de chacune d'elles toutes les instructions nécessaires pour répondre à la question ou pour quitter la page. Si les renseignements sont insuffisants, l'usager peut appuyer sur la touche HELP pour obtenir des explications supplémentaires. L'usager passe à 
une autre page lorsqu'il fournit une bonne réponse ou lorsqu'il appuie sur une touche fonction disponible à ce moment. Le passage d'une page à une autre s'effectue généralement en une fraction de seconde.

L'usager interroge les banques française ou anglaise en appuyant sur la touche LAB; il dactylographie ensuite le terme qu'il désire. Le système n'établit aucune distinction entre les majuscules et les minuscules, et ne tient pas compte des accents. Ainsi, les expressions «terminal PLATON» et «terminal platon», "entrée» et «entree» sont considérées comme identiques. Si le terme désiré n'existe pas dans la banque, le système en informe l'usager. S'il le désire, il peut appuyer sur la touche BACK pour consulter la liste des termes afin d'en choisir un autre ou il peut inscrire un commentaire. Si le terme choisi par l'usager a plus d'une traduction, le système lui demande d'en choisir une pour affichage. Ensuite, le système affiche une nouvelle page sur l'écran, et demande à l'usager s'il tient à consulter la définition, le contexte ou les notes concernant le terme choisi.

Lorsque l'usager a fait son choix, le système affiche encore une autre page contenant les renseignements demandés en offrant à la fois le contenu du terme choisi et celui de sa traduction dans l'autre langue. Ceci permet à l'usager de comparer les deux termes et de dégager les rapports existant entre eux. Ce procédé fait l'objet de la figure 1.A.

Cette façon de présenter les renseignements sur plusieurs écrans permet à l'usager d'obtenir seulement les renseignements dont il a besoin. Cela ne lui impose donc pas une quantité abondante de renseignements sur une même page. Ainsi, l'usager qui désire obtenir la traduction d'un terme en particulier n'a qu'à examiner la première page que le système lui présente.

Nous avons dit plus haut que le responsable est chargé de la mise à jour des banques française et anglaise. Ce travail lui est confié en propre pour deux raisons. Premièrement, toutes les données qui entrent dans la banque ont été soigneusement sélectionnées et normalisées pour maintenir dans les fichiers un haut niveau de qualité. Deuxièmement, la capacité de la mémoire auxiliaire est limitée et les ressources dont a besoin le responsable dépassent de loin celles d'un usager ordinaire. Comme le responsable peut, à son gré, ajouter, modifier ou supprimer les données, il est donc le principal «gardien» de la banque.

\section{L'EXPLOITATION}

En octobre 1979, PLATERM a pour la première fois fait partie d'un cours de traduction dispensé à l'Université du Québec à Trois-Rivières. Lors de cette expérience, vingt étudiants inscrits dans un cours d'informatique à l'intention des traducteurs se sont servis de PLATERM pendant une dizaine de semaines comme instrument de recherche pour réaliser divers travaux en traduction. Dans ces circonstances, PLATERM s'est substitué au dictionnaire traditionnel, bastion vénérable de la profession.

Le bilan de cette expérience se résume comme suit: des 357 entrées dans la banque anglaise et des 375 entrées dans la banque française, on a relevé en 
anglais 1728 accès à 348 termes et en français 732 accès à 357 termes. Le nombre étrangement élevé d'accès à la banque anglaise s'explique par le fait que tous les travaux allaient de l'anglais au français, d'où le besoin d'en savoir plus sur les mots anglais. Il va de soi que la traduction dans l'autre sens provoquerait le phénomène inverse. Enfin, les usagers se sont servis de PLATERM quelque 184 fois, soit un total de 38 heures.

À l'heure actuelle, PLATERM sert de façon quotidienne à trois traducteurs dont le mandat est de produire la version française du didacticiel ${ }^{9}$ du système américain PLATO.

Bientôt, à l'Université du Québec, on mettra des copies vierges du programme PLATERM à la disposition des autres projets de traduction touchant des domaines spécialisés de manière à accélérer, grâce à des bases de données automatisées, le processus habituellement lent de la traduction.

\section{LE VOCABUlAiRE PLATERM}

Dans la liste suivante, le lecteur remarquera que tous les termes anglais inscrits dans la banque sont accompagnés, à droite, de leur(s) traduction(s) française(s). Pour des raisons évidentes d'espace et d'intérêt, il n'est pas question de reproduire ici toute la banque, c'est-à-dire les définitions, les contextes ou exemples ainsi que les notes qui font partie de chaque entrée. Par contre, le lecteur saura qu'à l'égard de chacune de ces entrées, il trouvera dans la banque les renseignements complémentaires désirés.

ANGLAIS

Access

Access key

Account

Account director

Algorithm

Alternate font

Animated display

Animated sequence

Answer

Argument

Array

Arrow

Assign

Assignment

Assignment arrow

Attach

Attached unit

Author

Authoring

Author-type user

Author aids

Author language condenser

Author language instruction
Author language

\section{FRANÇAIS}

Accès
Touche accès
Compte
Coordonnateur des comptes
Algorithme
Jeu de caractères auxiliaire
Affichage animé
Séquence animée
Réponse
Argument
Tableau
Flèche
Affecter
Affectation
Flèche d'affectation
Raccorder
Élément raccordé
Médiatiseur
Médiatisation
Médiatiseur
Assistance au médiatiseur
Langage de médiatisation
Condenseur de langage médiatique
Instruction en langage médiatique

9. Didacticiel: (du grec didaskein, enseigner) ensemble de matériel didactique utilisant des ressources multimédias. 
Author language lesson

Author lesson

Author mode

Author mode display

Author user

Author vocabulary

Auxiliary unit

Backing out

Back out

Base pointer

Base sequence

Base unit

Bit

Bit off

Bit on

Blank tag

Block

Block list

Block listing display

Branch

Branching

Branching sequence

$B$ ranch instruction

Branch point

Byte

Cabling

CAI (Computer-assisted instruction)

Calculational command

Calculational loop

Calculational looping

Calc feature

Carriage return

Catalog

Catalogue

Catchall

CBE (Computer-based education)

Central processing unit

Change code

Character set

Clear

Clearing

Coarse grid

Code

Coding

Command

Command field

Comment feature

Common variables

Communication link

Computer-assisted instruction

Computer-based education

Computer-managed instruction

Computer game

Computer interface unit

Computer word

Condense

Condensing

Conditional branch

Conditional branching

Conditional branch instruction

Conditional command

Conditional operation
Leçon en langage médiatique

Leçon

Mode médiatique

Affichage du mode médiatique

Médiatiseur

Vocabulaire de médiatisation

Élément auxiliaire

Sortie forcée

Sortir de force

Pointeur d'élément nodal

Séquence nodale

Élément nodal

Bit

Bit à zéro

Bit à un

Référent vide

Bloc

Liste des blocs

Affichage de la liste des blocs

Aiguiller

Aiguillage

Séquence d'aiguillage

Instruction d'aiguillage

Point de branchement

Multiplet

Câblage

Apprentissage assisté par ordinateur

Commande de calcul

Boucle de calcul

Calcul en boucle

Fonction calc

Retour de chariot

Répertoire

Répertoire

A tout faire

Apprentissage par didacticiel

Unité centrale de traitement

Clé de modification

Jeu de caractères

Effacer

Effacement

Grille large

Codage

Codage

Commande

Champ commande

Fonction commentaire

Variables collectives

Lien de communication

Apprentissage assisté par ordinateur

Apprentissage par didacticiel

Gestion informatique de l'apprentissage

Jeu informatisé

Interface de réseau

Mot machine

Condenser

Condensation

Branchement conditionnel

Aiguillage conditionnel

Instruction conditionnelle d'aiguillage

Commande conditionnelle

Opération conditionnelle 
Constant

Consult feature

Conversational mode

Core

Core storage

Core store

Course

Courseware

Course director

CPU (Central processing unit)

Curriculum

Cursor

Cursor feature

Datafile

Dataset

Dataset file

Data bank

Data base

Data collection

Declaration

Defined variable

Dialogue

Dialogue mode

Dial up

Direct access

Direct access memory

Disable

Disk pack

Display

Display command

Display unit

Drill and practice

Dummy argument

Dummy tag

ECS (Extended core storage)

Edit

Editing

Editor

Embed

Embedded command

Embed symbol

End lesson unit

Erase

Erasure

Execution

Execution sequence

Extended core storage

Falling through

Fine grid

Finish unit

Flag

Floating comma representation

Floating point representation

Flowchart

Flow diagram

Font

FONT key

Force

Forced branching

Function key

Games

Grid
Constante

Fonction consultation

Mode dialogué

Tore

Mémoire à tores

Tore

Collège

Didacticiel

Régisseur

U.C.T. (Unité centrale de traitement)

Cheminement

Curseur

Fonction curseur

Fichier cumulatif d'apprentissage

Fichier de données

Fichier de données

Banque de données

Base de données

Saisie de données

Déclaration

Variable définie

Dialogue

Mode dialogue

Commuté

Accès direct

Mémoire à accès direct

Inhiber

Chargeur de disque

Affichage

Commande d'affichage (absolu)

Visuel

Exercices répétés

Référent fictif

Référent bidon

M.A. (Mémoire auxiliaire)

Éditer

Édition

Éditeur

Insérer

Commande d'insertion

Symbole d'insertion

Élément de fin de leçon

Effacer

Effacement

Exécution

Séquence d'exécution

Mémoire auxiliaire

Passage direct

Grille fine

Élément de sortie

Indicateur

Représentation en virgule flottante

Représentation en point flottant

Organigramme

Organigramme

Fonte

Touche FONT

Forcer

Aiguillage forcé

Touche fonction

Jeux

Grille 
Grid feature

Group

Group note

Hardware

Hard-wired

Help

Help-type key

Help sequence

Help unit

Ignorable word

Increment

Incremental

Index

Index expression

Individualized instruction

Initialization

Initialize

Initial entry unit

Input

Inquiry mode

Inspect code

Inspect mode

Instruction

Instructional lesson

Instructional material

Instructor

Integer

Integer representation

Integral number

Interact

Interactive

Interactive lesson

Interactive mode

Interface

Iteration

Iteration loop

I.E.U. (Initial entry unit)

Judge

Judging

Judging command

Judging copy

Judging instruction

Judging option

Judging state

Jump

Key

Keyboard

Keypress

Keyset

Keyword

Label

Learning

Lesson

Lesson data

Lesson execution

Lesson material

Lesson segment

Lesson sequence

Lesson space

Lesson testing

Line drawn character

List
Fonction grille

Collège

Note collective

Matériel

Câblé

Aide

Touche d'aide

Séquence d'aide

Élément d'aide

Mot négligeable

Incrément

Incrémentiel

Index

Expression d'index

Apprentissage individualisé

Initialisation

Initialiser

Élément initial d'entrée

Entrée

Mode questionnement

Clé de lecture

Mode de lecture

Instruction

Leçon d'apprentissage

Didacticiel

Moniteur

Entier

Représentation en nombres entiers

Entier

Dialoguer

Interactif

Leçon interactive

Mode dialogué

Interface

Itération

Boucle d'itération

E.I.E. (Élément initial d'entrée)

Évaluer

Evaluation

Commande d'évaluation

Copie d'évaluation

Instruction d'évaluation

Option d'évaluation

État d'évaluation

Sauter

Touche

Clavier

Frappe

Clavier

Mot clé

Étiquette

Apprentissage

Leçon

Notice de leçon

Exécution de la leçon

Didacticiel

Segment de leçon

Séquence d'une leçon

Plage

Vérification de la leçon

Caractère tracé par segments

Liste 


\begin{tabular}{|c|c|}
\hline Listing & Listage \\
\hline Literal & Littéral \\
\hline Load & Charger \\
\hline Loading & Chargement \\
\hline Logging in & Entrée \\
\hline Logging out & Sortie \\
\hline Logical operation & Opération logique \\
\hline Logical operator & Opérateur logique \\
\hline Logic operation & Opération logique \\
\hline $\log$ in & Entrer \\
\hline Log out & Sortir \\
\hline Loop & Boucle \\
\hline Looping structure & Structure en boucle \\
\hline Lowercase & Bas de casse \\
\hline Machine word & Mot machine \\
\hline Main memory & Mémoire centrale \\
\hline Main unit & Élément principal \\
\hline Mask & Masque \\
\hline Mass storage & Mémoire de masse \\
\hline Match & Concorder \\
\hline Match & Concordance \\
\hline Memory block & Bloc de mémoire \\
\hline Microfiche & Microfiche \\
\hline Microtable & Microtable \\
\hline Micro character & Caractère micro \\
\hline Mode & Mode \\
\hline Modulo & Modulo \\
\hline Monitor mode & Mode moniteur \\
\hline Multiple & Étudiant hors cadre \\
\hline Multiplexing & Multiplexage \\
\hline Nested loops & Boucles emboîtées \\
\hline NEXT key & Touche NEXT \\
\hline Notes feature & Fonction notes \\
\hline Number field & Champ numérique \\
\hline Operand & Opérande \\
\hline Output & Sortie \\
\hline Panel & Écran \\
\hline Password & Mot de passe \\
\hline Personal note & Note personnelle \\
\hline Plasma & Plasma \\
\hline Plasma display panel & Écran à plasma \\
\hline Plasma display screen & Écran à plasma \\
\hline PLATO & PLATON \\
\hline Plato author language & Langage de médiatisation \\
\hline Plato name & Nom d'usager \\
\hline Plato terminal & Terminal PLATON \\
\hline Pointer & Pointeur \\
\hline Primary sequence & Séquence principale \\
\hline Problem solving & Étude de cas \\
\hline Program & Programme \\
\hline Program loop & Boucle de programme \\
\hline Public note & Note publique \\
\hline Random & Aléatoire \\
\hline Randomly & De façon aléatoire \\
\hline Random access & Accès direct \\
\hline Random number & Nombre aléatoire \\
\hline Register & Inscrire \\
\hline Regular command & Commande d'exposition \\
\hline Regular font & Fonte principale \\
\hline Regular state & Etat d'exposition \\
\hline Relational operator & Opérateur de relation \\
\hline Relocatable display command & Commande d'affichage relati \\
\hline
\end{tabular}


Remedial

Required word

Reserved word

Response

Restart

Restart unit

Roster

Rotated writing

Route

Router

Router lesson

Sampling without replacement

Sampling with replacement

Scaling factor

Scanning

Screen

Security code

Self-pacing

Sequence

Shifted function key

Signing on

Sign off

Sign on

Sign on sequence

Simulation

Simulation mode

Site

Site controller

Site director

Sized writing

Software

Source code

Specification command

Statement

Station

Step mode feature

Step feature

Step mode

Storage block

Store

Storing

Student

Student-type user

Student mode

Student record

Student variable

Subscript

Subscription

Swap

Swapping

Sweeping

System

System announcement

System lesson

System programmer

System reserved word

System security

System variable

TAB key

Tag

Tag field

Talk feature
De rattrapage

Mot indispensable

Mot réservé

Réponse

Redémarrage

Élément de redémarrage

Liste

Écriture pivotée

Guider

Guide

Guide

Échantillonnage sans remplacement

Échantillonnage avec remplacement

Facteur d'échelle

Balayage

Écran

Clé de protection

Progression libre

Séquence

Touche-fonction majuscule

Entrée

Sortir

Entrée

Séquence d'entrée

Simulation

Mode simulation

Station

Contrôleur local

Proviseur

Caractère de taille variable

Logiciel

Code source

Commande de spécification

Énoncé

Poste

Mode pas à pas

Fonction pas à pas

Mode pas à pas

Bloc de mémoire

Emmagasiner

Emmagasinage

Étudiant

Étudiant

Mode étudiant

Dossier d'étudiant

Variable individuelle

Indice

Abonnement

Échanger

Échange

Balayage

Système

Avis système

Programme

Programmeur système

Mot réservé au système

Protection du système

Variable du système

Touche TAB

Référent

Champ référent

Fonction conversation 
Teaching

Time sharing

Time slice

Tolerence

Touch input

Touch panel

Tutorial mode

Tutor language

Union

Unit

Uppercase

User

Welcome page

Wired-in

Wiring

Word length

Word separator

Wrap around

Wrap round
Enseignement

Temps partagé

Tranche de temps

Tolérance

Entrée tactile

Écran tactile

Mode tutoriel

Langage PLATON

Union

Élément

Haut de casse

Usager

Page d'accueil

Câblé

Câblage

Longueur du mot

Séparateur de mots

Report circulaire

Report circulaire

FIGURE 1.A

Quel terme voulez-vous?

lesson (Réponse de l'usager)

Traduction(s):

1. 1 econ

2. programme

Quel le traduction voulez-vous?

\$ (L'usager inscrit 1 et le système affiche la figure l.B)

-banque anglaise-

- faites HELP pour information.

- faites SHIFT-STOP pour sortir.

- faites LAB pour changer de banque.

- faites BACK pour une autre option. 
FIGURE 1.B

lesson (Terme anglais)

lecon (Traduction)

dernière modification

$16 / 22 / 79$ dernier acces

03, 30.80

(Statistiques)

Voulez-vous consulter:

nombre d'acces

1. les notes

* (Avertissement)

25

2. 1 'exemple

10

3. Ia définition

16

\% (L'usager inscrit 3 et le système affiche la figure I.C)

-DFTf pour statistiques-

(L'usager a appuyê sur cette touche)

- faites HELP pour information.

- faites SHIFT-STOP pour sortir.

- faites BACK pour un autre terme.

-raites SHIFT-BACK pour une autre option.

FIGURE 1.C

lesson

Définition:

(n.) Term referring to the lesson, i.e. courseware that the author has composed, or to the lesson, i.e. a computerized entity to be executed. 
FIGURE 1.C (stite)

1 econ

Définition:

(n.f.) Selon le cas, désigne le matériel pedagogique, ou plus souvent les programmes qui en sont la

version médiatisés. (CoT)

(En appuyant sur DATA et en inscrivant COT, l'usager voit au bas de l'écran le titre de l'ouvrage).

(Si l'usager appuie sur BACK, le systême affichera la figure l.B).

- faites HELP pour information.

\section{BIBLIOGRAPHIE}

BERGERON, V., Burke, D. C., en collaboration, Lexicographie, bilinguisme, juridique et ordinateur, Les Éditions de l'Université d'Ottawa, Ottawa, 1976.

BITZER, D. L., et al, Computer-based science education, CERL Report X-37, Computer-based Education Research Laboratory, University of Illinois, Urbana, 1973.

CDC, Plato Author Language Reference Manual, Publication No. 97405100, Control Data Corporation, St. Paul, Minnesota, 1977.

CDC, Plato System Overview, Publication No. 97406700, Control Data Corporation, St. Paul, Minnesota, 1977.

CDC, Plato User's Guide, Publication No. 97405900, Control Data Corporation, St. Paul, Minnesota, 1976.

CHOUINARD, R., PLATON, un système multimédia d'apprentissage, information générale 2, publication no Pl. 2A, Université du Québec, Québec, 1977.

DUBUC, R., Manuel pratique de terminologie, Linguatech, Montréal, 1978.

DUBUC, R., "Les textes traduits et leur utilisation en terminologie», Meta, Journal des traducteurs, 19, 205-208 (1974).

DUBUC, R., Banque de terminologie de l'Université de Montréal, Description du système TERMIUM, Université de Montréal, Montréal, 1975.

GHESQUIËRE, J., C. DAVIS et C. THOMPSON, Introduction to Tutor, Computer-based Education Research Laboratory, University of Illinois, Urbana, 1974.

MALCHONOVA, T. V., Maintaining information retrieval thesauri, Automatic Documentation and Mathematical Linguistics, 11, 20-27 (1977).

OFFICE DE LA LANGUE FRANÇAISE, Les données terminologiques, actes du colloque international de terminologie, Éditeur officiel, Québec, 1975.

PLATO IV, Software Group, PLATO IV, Authoring, Int. J. Man-Machine Studies, 6, 445-463 (1974).

SCHULZ, J. et H. GÖRICKE, The Dictionary in the Computer, Possibilities of Directly interrogating a Multilingual Terminology Data Bank via Video Display Units, Babel, 23, $29-33$ (1977). 